\title{
A Mechatronic Approach for the Detection of Wheel Slip/Slide and Antislip Control of Locomotive with AC Traction Motors
}

\author{
Branislav Gavrilovic \\ Railway College of Vocational Studies, Zdravka Celara, Belgrade, Serbia
}

\section{Email address:}

gavrilovicbranislav5@gmail.com

\section{To cite this article:}

Branislav Gavrilovic. A Mechatronic Approach for the Detection of Wheel Slip/Slide and Antislip Control of Locomotive with AC Traction Motors. American Journal of Mechanics and Applications. Vol. 5, No. 6, 2017, pp. 47-52. doi: 10.11648/j.ajma.20170506.11

Received: November 27, 2017; Accepted: January 15, 2018; Published: January 25, 2018

\begin{abstract}
This paper presents a mechatronic approach for the detection of wheel slip/slide and antislip control of locomotive with ac traction motors, to enable an optimal use of adhesion in poor contact conditions. The proposed technique explores the variations in wheelset dynamic properties caused by condition changes at the wheel-rail contact and detects slip conditions from measurement of train speed with internal optical encoder of the wheelset axle indirectly. The modeling of a typical traction system, consisting of an induction traction motor (with associated power inverter and field-orientated control) connected to a wheelset via a gearbox, is introduced. The development of the slip detection and control scheme is presented, and the effectiveness of the proposed technique is demonstrated using computer simulations.
\end{abstract}

Keywords: Slip-Slide Control System, Adhesion Coefficient, Anti-Slip Drive

\section{Introduction}

Serbian Railways (ŽS) has ordered 21 electric FLIRTs (Fast Light Innovative Regional Train) from Stadler Rail for commuter rail services in the Belgrade region. This generation electric multiple unit FLIRTs use AC motors for wheel set traction motor that are significantly more reliable and unsophisticated if compared to the previous DC motors used for drives. The three-phase asynchronous traction motor is the motor used most frequently for modern locomotive drives because of its excellent reliability, its simple and thus low-cost construction and low maintenance costs. The threephase asynchronous traction motor is also known as squirrelcage rotor. On the other hand, $\mathrm{AC}$ motor rotation speed adjustment differs essentially from DC motor rotation speed change. Thus, aiming to control and adjust slip and slide processes of drive wheel sets it is necessary to apply different principles of electrical machine operation parameter tracking, measurement and adjustment.

A appearance and principal sketch of this type of vehicle showing its main electrical components is given in Figure 1.

There is a line side and motor side of the intermediate DClink. The vehicle's interface to the rest of the power system is a voltage-source converter (VSC), mentioned as the 'lineside converter', behind a step-down transformer. The lineside converter's task is to supply the DC-link capacitance with energy from the overhead contact line in order to keep the DC-link voltage constant. The DC-link capacitance is the energy source for the motor VSC, often called the motor-side inverter, and the induction motor.

For high-power locomotives, Gate Turn-Off (GTO) thyristors as semiconductors has been common until recently, but the use of Insulated-Gate Bipolar Transistors (IGBT) is increasing as the development proceeds. In order to reduce harmonics the semiconductors are commonly switched with a pulse-width modulated (PWM) pattern.

The line-side converter is able to operate in all four quadrants, independently controlling active and reactive power. Great features of this technology are the possibility for feeding power back into the network when regenerative braking and improving active power flow by inductive and capacitive reactive power. The line-side converter is able to operate in all four quadrants, independently controlling active and reactive power. Great features of this technology are the possibility for feeding power back into the network when regenerative braking and improving active power flow by 
inductive and capacitive reactive power.

Control of these systems is complex but it is all carried out by microprocessors. The control of the voltage pulses and the frequency has to be matched with the motor speed. The changes which occur during this process produce a set of characteristic buzzing noises which sound like the "gear changing" of a road vehicle and which can clearly be heard when riding on the motor car of an $\mathrm{AC}$ driven electric multiple unit (EMU).
The locomotive wheel set slippage is evident when the locomotive traction force exceeds adhesion force. The main locomotive traction law is the following [1]:

$$
\mathrm{F}_{\mathrm{TR}} \leq \psi_{\mathrm{ath}} \cdot \mathrm{P}_{\mathrm{ath}}
$$

where: $\psi_{\text {ath }}-$ locomotive wheel-sets' adhesion to rail coefficient; $P_{a t h}$ - locomotive adhesion weight.
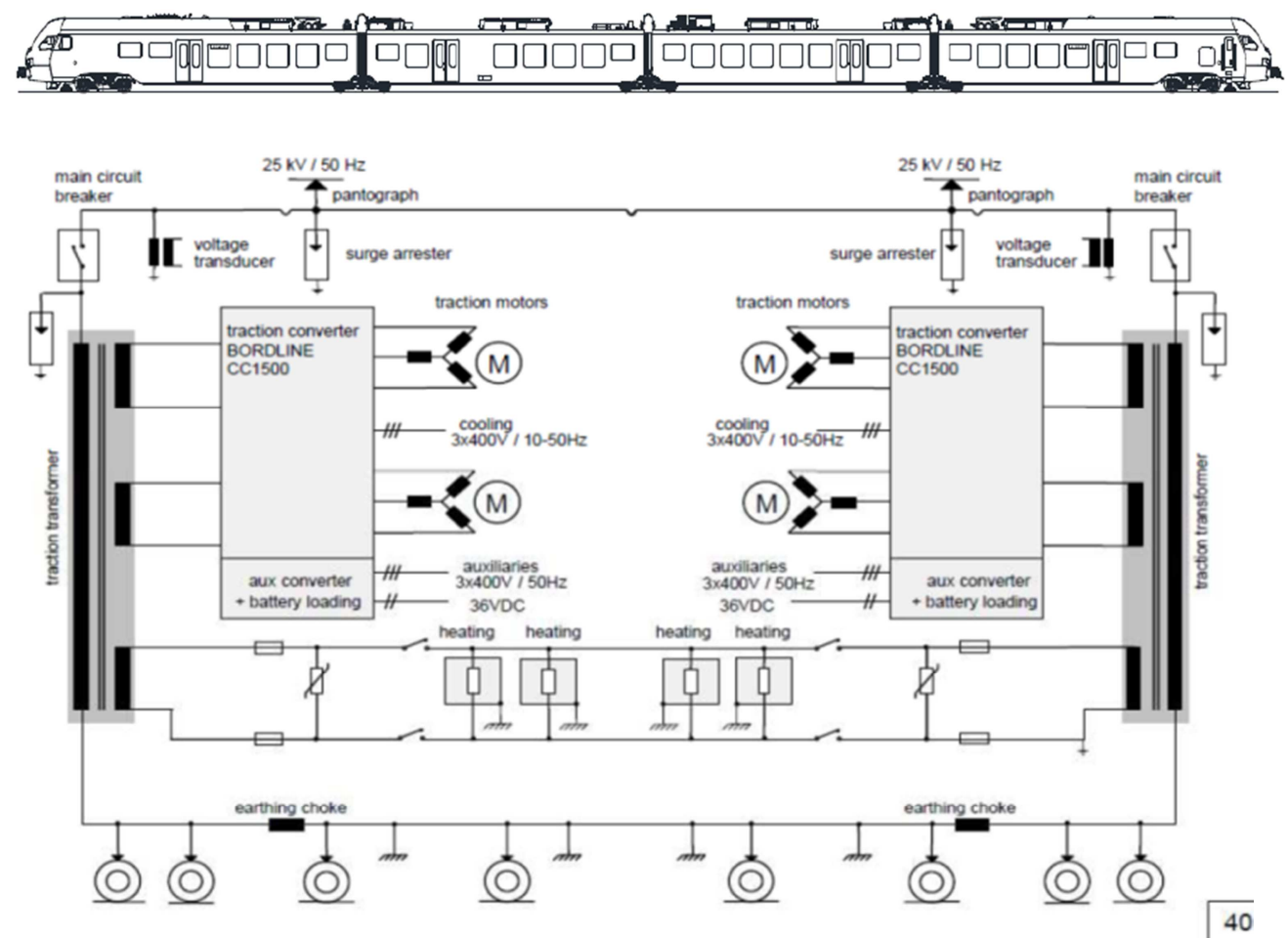

Figure 1. A appearance and principal sketch of the electric FLIRTs from Stadler Rail.

In order to maintain stable traction mode (to avoid wheels slip) it is necessary to maintain formula (1) inequality condition. The change of locomotives wheel-sets adhesion coefficient value in different seasons is provided in figure 2 .

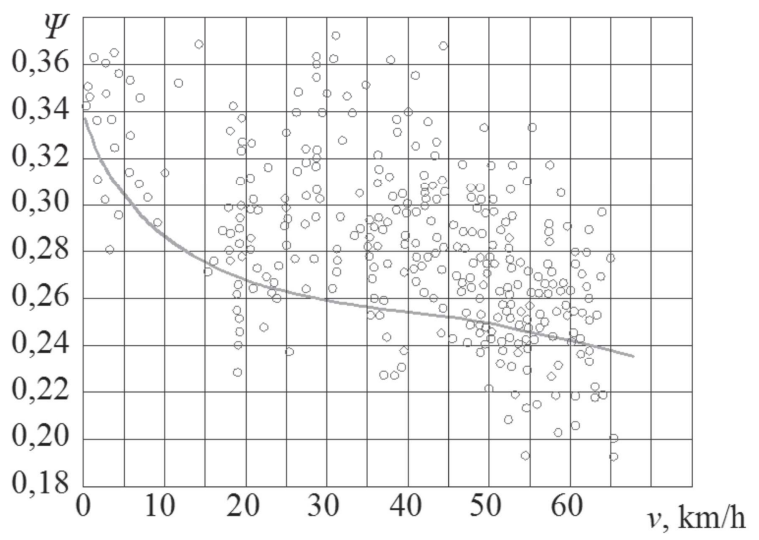

Figure 2. Locomotive adhesion coefficient dissipation dependence on locomotive speed in different seasons.

The estimation of adhesion coefficient of a newly made electric FLIRTs is carried out by means of practical tests in different seasons and different day time. In order to obtain objective results several hundred of practical tests are carried out. Figure 2 shows that adhesion coefficient values are highly dissipated as there are many factors that influence in case of equal train mass [2].

The electric FLIRTs adhesion coefficient value in the season change time (dissipated, e.g. 0.33 to 0.2 ) is as shown in figure 2. However, when operating the said electro motor unit in season change time, the rolling stock mass is not adjusted due to the changes of adhesion conditions. Caused by the decrease of the adhesion coefficient the traction power becomes insufficient for this rolling stock towing. In such a case sand is sprinkled intensively to increase the adhesion coefficient. However electro motor unit starting traction time is increased due to intensive slipping, thus causing accelerated wheel set and rail wear.

The article discusses theoretical and practical aspects of formation of wheel set rolling surface conicality, rail tilt angle and wheel pair position in the track, redistribution of electric multiple unit drive wheel set axis force to rail in traction mode at slip conditions.

There are analyzed peculiarities of locomotive adhesion 
weight optimum usage, axis force change in traction mode. The influence of adhesion ratio change to traction characteristic and traction force change are described. There are examined various electric multiple unit construction schemes with AC traction motor drive.

\section{Automatic Control of Slipping Process of Locomotives with AC Traction Motors}

Figure 3 shows automatic wheel-sets anti-slip and slide control system that is proposed by the authors for computer aided control of locomotive wheel set slipping and sliding process that is comprised: wheel set speed sensors BR1- BR6 that are mounted in traction motors, A and B bogie wheel set speed tolerance signal comparison blocks SCBI, SCBII [3]. When tolerance of one of individual wheel sets of bogies (A or $\mathrm{B}$ ) is exceeded, there are generated control signals $\mathrm{A}$ and
B that adjust signals sent by computer aided control system (in Figure 3 marked respectively as XA and $\mathrm{XB}$ ) to inverter elements IGBT transistors that have changing on and off cycles.

The automatic control system of slipping process that is proposed by the authors shall decrease the value of supply power and its frequency of one bogie from the two asynchronous engines for a short time. Thus the rotation speed of one bogie of traction motor-wheel set shall be equalized and wheel to rail adhesion shall be restored. Such dynamic characteristics are shown in figure 4 in green as E1Y1 with supply power frequency f1(1). E2-Y2 at supply power frequency f1(2), E3-Y3 with supply power frequency $\mathrm{fl}(3)$. When adhesion conditions are restored, there is automatic restoration of ATM supply power and its frequency value, i.e. it returns to any point of the previous operating characteristic (figure 4). Traction forces are not disrupted in locomotive control process as slipping process is controlled automatically.

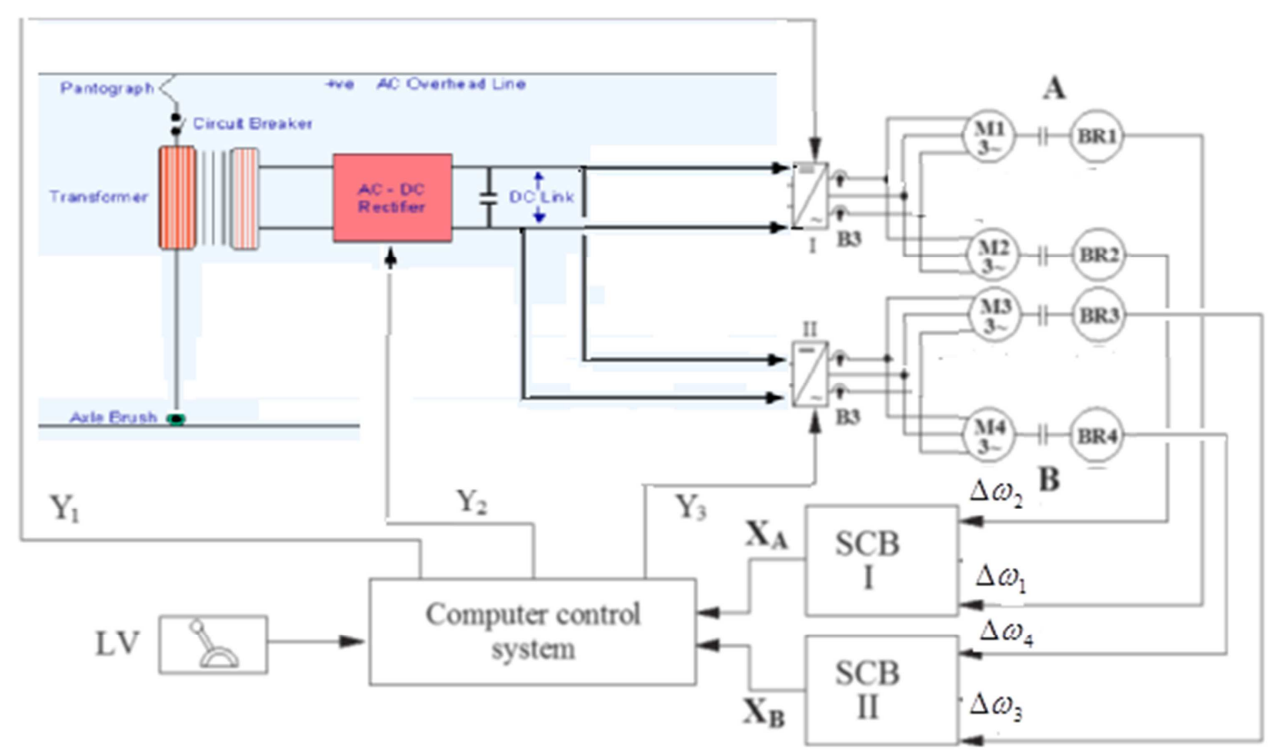

Figure 3. Circuit diagram of electro locomotive with AC traction motors automatic wheel-sets anti-slip and slide control system process parameters computer drive: SCBI-traction motors speed control block (bogie A); SCBII - traction motors speed control block (bogie B).

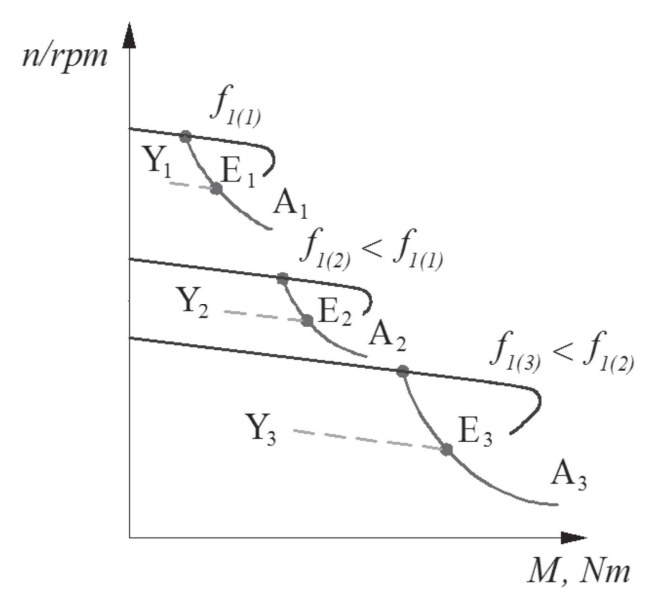

Figure 4. Artificial asynchronous mechanical characteristics of asynchronous motor when ATM frequency rotation speed control method is applied.

\section{Algorithm for Slipping Process Parameter adjustment and Control}

The authors provide computer aided control system of locomotive wheel set slipping and sliding process control. Variants of the control algorithm: a - wheel set speed tolerance signals are compared with the tolerance signals in blocks SCBI, SCBII and they are adjusted by changing parameters of that inverter to control signals $\mathrm{Y} 1$ and $\mathrm{Y} 3$ (thereof the respective two asynchronous traction engines (individual controlled) are powered). b - in case with controlled parameters of inverters I and II the process of bogie wheel set slippage is continued and bogie wheel set speeds are not equalized, the generator voltage of synchronous traction motor is limited by decreasing excitation current with signal Y2 (general parameter control). 
Computer aided control system of electro locomotive wheel set slipping-sliding process consists (figure 3): M1,.., M4 - asynchronous traction motors; speed sensors of BR traction motors; LV - locomotive driver; SCBI - bogie A wheel set speed tolerance signal comparison unit; SCBII- Bbogie wheel set speed tolerance signal comparison unit; Y1 control signal that adjusts inverter I electrical parameters; Y2 - synchronous traction motor signal that adjusts AC-DC recifer; Y3 - control signal that adjusts inverter II electrical parameters; $\mathrm{R}$ - excitation current regulator of synchronous traction generator; $\Delta \omega 1, \ldots, \Delta \omega 4-$ speed tolerance signals of traction motor; XA - total compared bogie A wheel set speed tolerance signal; XB - total compared bogie B wheel set speed tolerance signal.

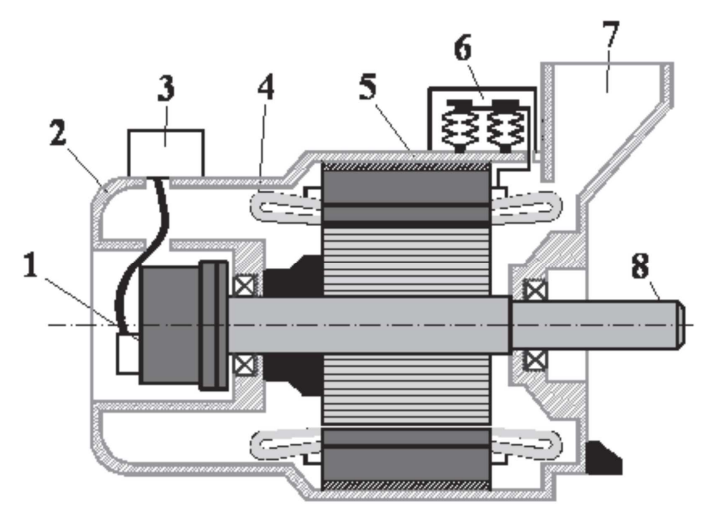

Figure 5. Asynchronous traction motor with internal optical encoder: 1encoder; 2 -stator; 3 -terminal box; 4 -stator windings; 5 -ferromagnetic core; 6-clamps; 7 - cooling channel; 8 -shaft.

Authors propose to install an encoder in ATM rotation speed and location coordinate measurement sensors BR that are installed in the traction motor (see figure 5). The encoder is analogous or digital converter with analogous signal at the output or a certain number of pulses that is proportional to rotation speed or turn angle [4].

To use an encoder in ATM vector control system is suggested in literature $[1,2,3]$. The encoder consists of a light source, mask, code disk and sensors. The code disc contains artificial spaces that make light permeable and impermeable segments. Light sensitive sensors are mounted behind the code disc. The scheme of optical encoder is provided in figure 6 [4].

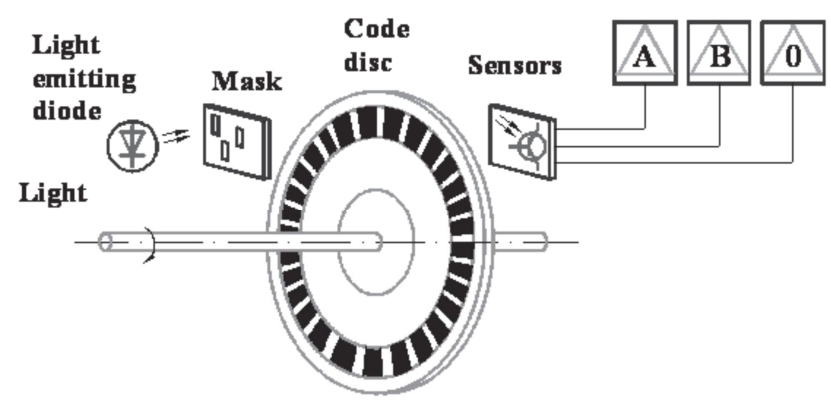

Figure 6. Optical encoder scheme.

Railway vehicle slip can be detected as shown in Figure 7.

Proposed control strategy require the detection method (a) by dividing vehicles into existing line and high speed line, and comparing speed of four axles and vehicle speed transmitted by communications, and by setting detection reference speed $v_{d m}$.

(a) slip speed $v_{s} \geq$ detection reference value $v_{d m}$

(b) motor acceleration $\alpha_{M} \geq$ detection reference value $\alpha_{d m}$

(c) slip acceleration $\alpha_{S} \geq$ detection reference value $\alpha_{d m}$.

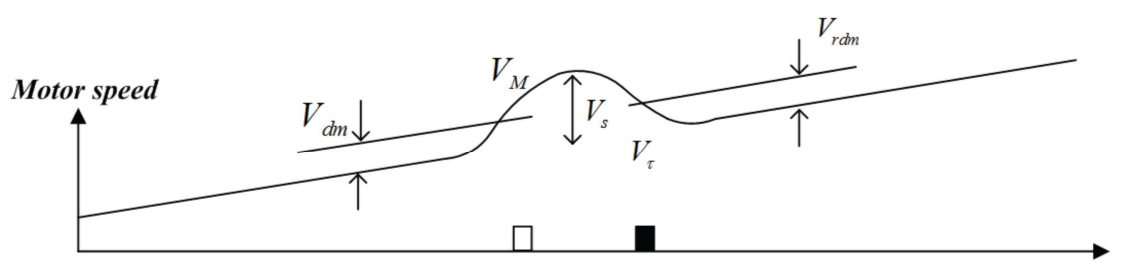

(a)

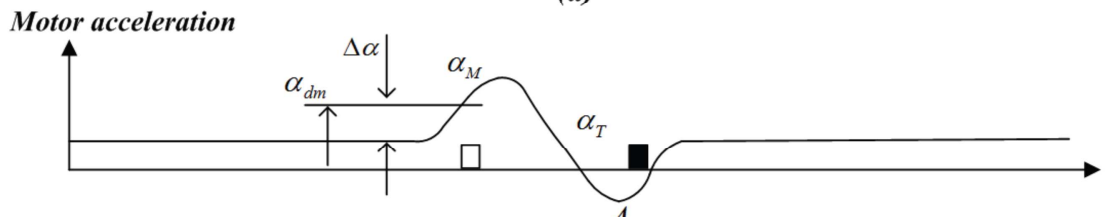

(b) ${ }^{A}$

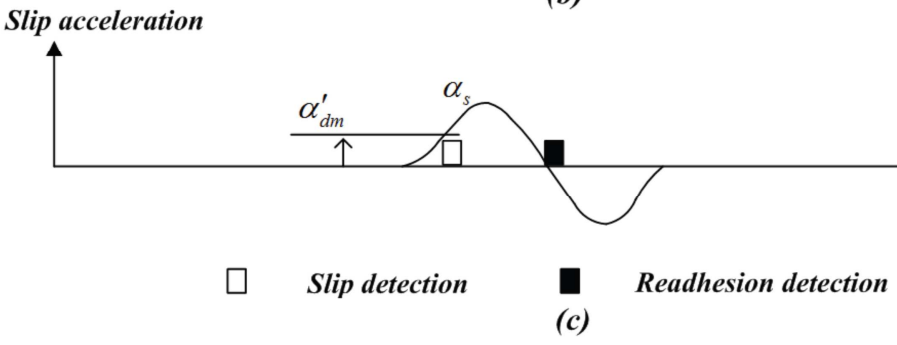

Figure 7. Detection methods of re-adhesion and slip. 
If slip speed is faster than detection reference value, it is possible to detect stably about changing environment. But rapid control is impossible because of slow detection. In the case of (a), though slip detection is stable, detection is slow. In the cases of (b) and (c), detection speed is faster than the case of (a). But, since they are sensitive to small changes of motor speed, being unstable to system disturbances, for complemental purposes, it is necessary to use low-pass filter component of acceleration speed as slip detection.

When encoder is installed in the ATM control system that is proposed by the authors, there can be received analogous or digital information, thus developing analogous-digital (hybrid) locomotive electrical drive control system [5].

All of them are suitable for computer system of electro locomotive wheel set slipping-sliding process control that is proposed by the authors.

\section{Tests of Locomotive Wheel Set Slipping and Sliding Process Computer Aided Control System in Different Conditions}

The proposed algorithm for slipping process parameter adjustment and control ensures highly efficient wheel-to-rail force transmission by means of continuous wheel force at its rim; slip control; limitation of wheel acceleration; reliable determination of the reference speed, which should represent the true train speed.

The algorithm protects the mechanical components against excess stress and reduces wear on the rails and wheel-set by avoiding: wheel blocking (flat spots on the running surface); synchronous drifting of the wheel speeds (worn rail heads); inherently stable rotational vibrations in the drive train.

The proposed algorithm continuously monitors the movement of the vehicle and the running wheels. If the movement variables deviate from the tolerance values, the tractive effort demanded by the overall control level is automatically reduced to a level which can be physically transmitted from the wheel to the rail.

Due to continuous monitoring of the movement variables relating to the vehicle and the wheels, it is ensured that traction is kept under control under different track conditions.

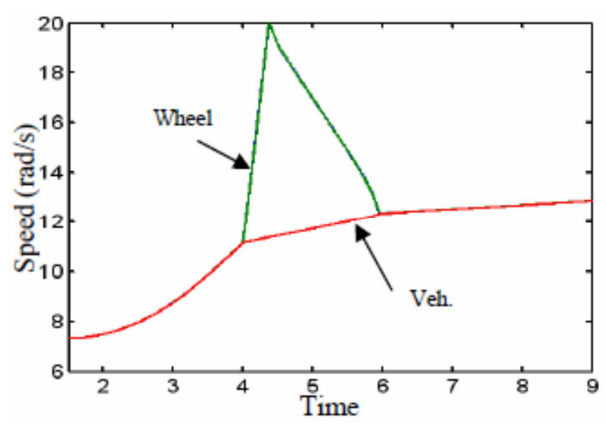

Figure 8. Adhesion control - reduced adhesion at $t=4 \mathrm{~s}$ (initial vehicle speed of $10 \mathrm{~km} / \mathrm{h}$ ).
The worst case scenario for wheel slip is if there is a sudden reduction of adhesion on the track when there is a high level of tractive effort applied. Figure 8 compares wheel and (equivalent) vehicle angular velocities during the vehicle acceleration from an initial speed of $10 \mathrm{~km} / \mathrm{h}$. At the time $\mathrm{t}=$ $4 \mathrm{~s}$, the adhesion of the track is reduced to well below the tractive effort, and consequently a severe wheel slip occurs and the slip ratio reaches as much as $60 \%$ in less than $0.5 \mathrm{~s}$ before being detected. The re-adehsion control is clearly effective in reducing the torque output from the motor rapidly and the recovery time takes about $1.5 \mathrm{~s}$.

If the adhesion is already low when a tractive effort is applied and increased gradually, the wheel slip (when it occurs) tends to be less severe as the net torque driving on the wheelset is relatively low. On the other hand the torsional vibrations will also be smaller and less sensitive to slip conditions. However, the proposed control scheme appears to deliver a robust performance in different conditions as illustrated in Figures 9 and 10. At the low (initial) speed of $10 \mathrm{~km} / \mathrm{h}$, the peak slip ratio reaches approximately $20 \%$ and the wheel slip is quickly detected and a complete adhesion is achieved within a fraction of a second. At the higher speed of $100 \mathrm{~km} / \mathrm{h}$, the peak slip ratio is about $14 \%$ but the recovery time takes somewhat longer as the system has to overcome a higher level of kinematic energy stored in the wheelset.

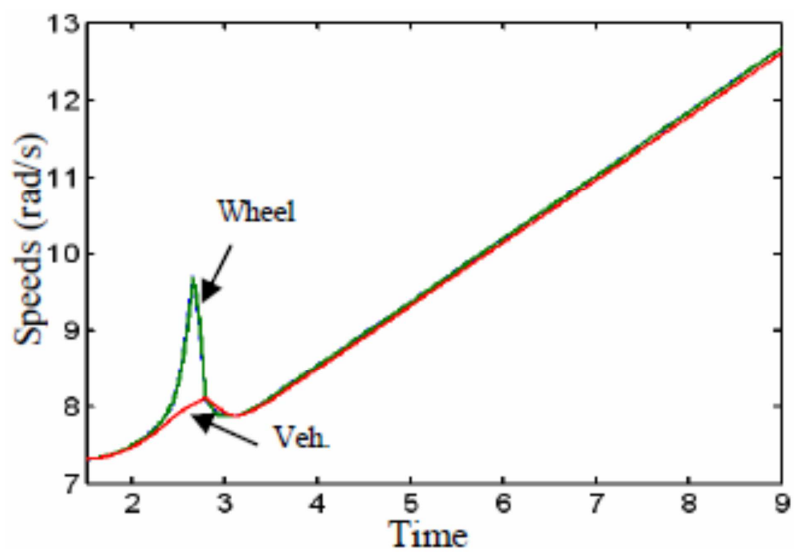

Figure 9. Adhesion control - low adhesion from $t=0 \mathrm{~s}$ (initial vehicle speed of $10 \mathrm{~km} / \mathrm{h}$ ).

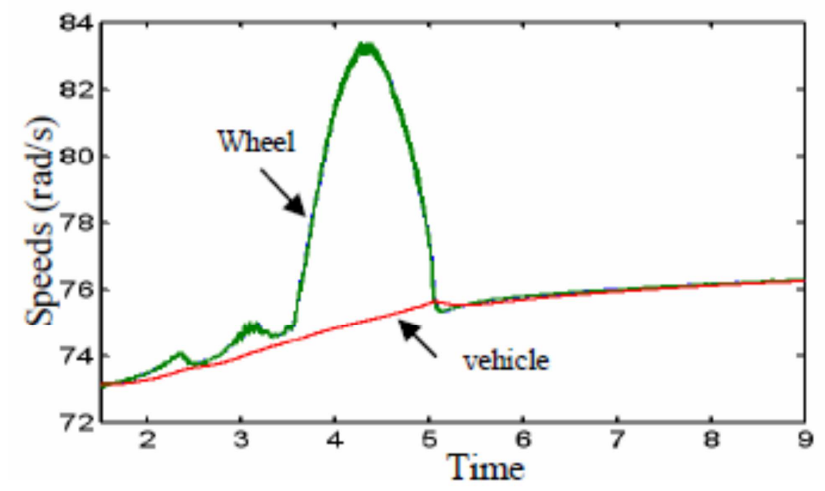

Figure 10. Adhesion control - low adhesion from $0 \mathrm{~s}$ (initial vehicle speed of $100 \mathrm{~km} / \mathrm{h}$ ). 
Figure 11 shows the simulation results using a more rigid axle where the torsional frequency is changed from 60 to 80 $\mathrm{Hz}$. Again the detection of a wheel slip and a complete adhesion are achieved in less than 2 s. In this, a second (smaller) wheel slip is observed which is quickly detected and eliminated by the controller - a clear evidence that the system operates very closely to the maximum adhesion region on the creep-creep force curve which is expected.

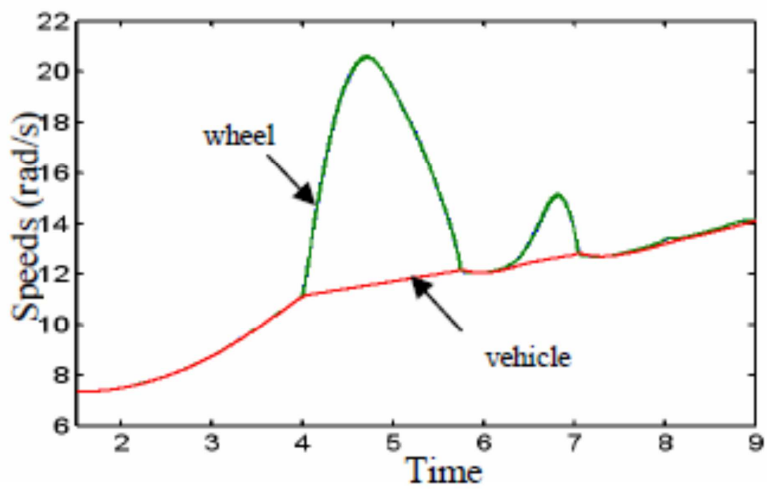

Figure 11. Reduced adhesion at $t=4 \mathrm{~s}$ (a more rigid axle, initial vehicle speed of $10 \mathrm{~km} / \mathrm{h}$ ).

\section{Conclusions}

1. The non-traditional computer aided slipping an sliding control system of locomotive AC traction motors that is proposed by the authors allows automatic continuous control of electrical parameters of the AC-DC recifer and the inverter in traction mode (dynamic mode) with the simultaneous slipping process control.

2. When the provided slip and slide control system of locomotives with AC traction motors is used, train driver does not need to interrupt traction mode control and this way there are no conditions for the formation of the rolling stock longitudinal tensile and compression forces.

3. When the provided slip and slide control system of locomotives with AC traction motors is used, there is optimal traction force control.

4. When the provided slip and slide control system of locomotives with AC traction motors is used, the adhesion coefficient is little dependent on the season.
5. When the provided slip and slide control system of locomotives with AC traction motors is used, the adhesion coefficient is recovered automatically and there is no need to put sand under the wheels to increase the adhesion.

\section{References}

[1] L. P. Lingaitis and L. Liudvinavičius, "Electric drives of traction rolling stocks with AC motors", Transport, vol. 21(3): pp. 223-229, 2006.

[2] K. Kondo, "Anti-slip control technologies for the railway vehicle traction", Vehicle Power and Propulsion Conference (VPPC), IEEE, no., pp.1306-1311, 2012.

[3] D. Frylmark, "Automatic Slip Control for Railway Vehicles", M. S. thesis, Dept. of Elect. Eng., Linkopings univ., Linkopings, Sweeden, 2003.

[4] D. Atmadzhova, "A method for determination of the wheel rail contac", Proceedings of the XII Railcon' 06 - ScientificExpert Conference on Railways, pp 33-36, Nis, Serbia, 2006.

[5] P. Pichlik, "Hybrid Slip Control Method for Railway Traction", Processing of the $17^{\text {th }}$ International Student Conference on Electrical Engineering - POSTER 2013, Prague, 2013, pp.35-41, 2013.

[6] H. Park, J. Kim, J. Choi and H. Yamazaki, H.; "Modeling and control of adhesion force in railway rolling stocks," Control Systems, IEEE, vol. 28, no. 5, pp.44-58, October 2008.

[7] T. Watanabe, "Anti-slip Readhesion Control with Presumed Adhesion Force. Method of Presuming Adhesion Force and Running Test Results of High Speed Shinkansen Train," Quarterly Report of Railway Technical Research Institute, QR of RTRI, vol. 41, No. 1, Mar 2000.

[8] X. Mei, H. Yu and A. Wilson, "A Mechatronic Approach for Anti-slip Control in Railway Traction", Proceedings of the $17^{\text {th }}$ World Congress, The International Federation of Automatic Control, Seoul, Korea, July 2008.

[9] M. Spiryagin, S. Lee and H. Yoo, "Control system for maximum use of adhesive forces of a railway vehicle in a tractive mode," Mechanical Systems and Signal Processing 22, pp 709- 720, 2008.

[10] X. Mei, H. Yu and A. Wilson, "Wheelset dynamics and wheel slip detection", STECH 2006, Chengdu, China. 2006. 